\title{
Effectiveness of lower target temperature therapeutic hypothermia in post-cardiac arrest syndrome patients with a resuscitation interval of $\leq 30 \mathrm{~min}$
}

Tadashi Kaneko $^{1 *}$, Shunji Kasaoka', Takashi Nakahara ${ }^{2}$, Hirotaka Sawano ${ }^{3}$, Yoshio Tahara ${ }^{4}$, Mamoru Hase ${ }^{5}$, Kenji Nishioka ${ }^{6}$, Shinichi Shirai ${ }^{7}$, Hiroshi Hazui ${ }^{8}$, Hideki Arimoto ${ }^{9}$, Kazunori Kashiwase ${ }^{10}$, Tomokazu Motomura ${ }^{11}$, Yasuhiro Kuroda ${ }^{12}$, Yuji Yasuga ${ }^{13}$, Naohiro Yonemoto ${ }^{14}$, Hiroyuki Yokoyama ${ }^{4}$, Ken Nagao ${ }^{15}$, Hiroshi Nonogi ${ }^{16}$ and on behalf of the J-PULSE-Hypo investigators

\begin{abstract}
Background: Therapeutic hypothermia $(\mathrm{TH})$ is a standard strategy to reduce brain damage in post-cardiac arrest syndrome (PCAS) patients. However, it is unknown whether the target temperature should be adjusted for PCAS patients in different states.

Methods: Participants in the J-PULSE-Hypo study database were divided into lower (32.0-33.5 ${ }^{\circ} \mathrm{C}$; Group L) or moderate $\left(34.0-35.0^{\circ} \mathrm{C}\right.$; Group M) temperature groups. Primary outcome was a favourable neurological outcome (proportion of patients with a Glasgow-Pittsburgh Cerebral Performance Category [CPC] of 1-2 on day 30). We compared between the two groups and in subgroups of patients divided by age and resuscitation interval (interval from collapse to return of spontaneous circulation) by propensity score (PS) analysis.

Results: Overall, 467 participants were analysed. The proportions of patients with favourable neurological outcomes were as follows (Group L vs. Group M) (OR; Odds ratio): all patients, $64 \%(n=42)$ vs. $55 \%$ ( $(n=424)$ (PS; OR 1.381 $(0.596-3.197)), P=0.452)$ and resuscitation interval $\leq 30 \mathrm{~min}, 88 \%(n=24)$ vs. $64 \%((n=281)$ (PS; OR 7.438 $(1.769-31.272)), P=0.007)$.

Conclusions: PCAS patients with a resuscitation interval of $<30$ min may be candidates for $\mathrm{TH}$ with a target temperature of $<34^{\circ} \mathrm{C}$.

Trial registration: University Hospital Medical Information Network (UMIN) Clinical Trials Registry UMIN000001935; available at: https://upload.umin.ac.jp/cgi-open-bin/ctr/ctr.cgi?function=brows\&action=brows\&type=summary\& recptno $=$ R000002348\&language $=J$.
\end{abstract}

Keywords: Target temperature, Rapid cooling, Prolonged hypothermia, Slow rewarming, Post-cardiac arrest syndrome

\footnotetext{
* Correspondence: kaneyui-ygc@umin.ac.jp

'Emergency and General Medicine, Kumamoto University Hospital, 1-1-1 Honjo,

Chuo-ku, Kumamoto 860-8556, Japan

Full list of author information is available at the end of the article
} 


\section{Background}

The efficacy of therapeutic hypothermia (TH) in postcardiac arrest syndrome (PCAS) patients was demonstrated by two, randomised controlled studies $[1,2]$. Current guidelines for resuscitation now recommend $\mathrm{TH}$ of $32-34{ }^{\circ} \mathrm{C}$ for $12-24 \mathrm{~h}$ in PCAS patients. However, a recent randomised controlled trial, the target temperature management (TTM) study [3], did not show any benefit of $\mathrm{TH}\left(33^{\circ} \mathrm{C}\right)$ for PCAS patients when comparing $\mathrm{TH}\left(33^{\circ} \mathrm{C}\right)$ and controlled normothermia $\left(36{ }^{\circ} \mathrm{C}\right)$. Therefore, there is still no clear evidence to show whether lower temperature treatment or antifever treatment should be considered for managing PCAS patients. Despite the limited clinical evidence, recent animal studies have shown beneficial effects of $\mathrm{TH}$ on neurological outcomes [4-6]. This raises the question of what causes the differences in the neurological outcomes between clinical and animal studies. Therefore, we hypothesised that there are some subgroups of patients in clinical settings who may show the greatest efficacy of lower target temperature TH.

The J-PULSE-Hypo study registry is a Japanese prospective cohort observational study of TH in PCAS patients. A total of 14 medical centres participated in this registry, which was originally conducted between January 2005 and 2009, and was then extended to March 2013 [7]. In the present study, we performed analyses of the J-PULSE-Hypo registry database to address our objective, which was to identify subgroups of patients who might be suitable candidates for lower target temperature $\mathrm{TH}$.

\section{Methods}

\section{Study design}

The present study used the J-PULSE-Hypo registry database, a prospective cohort database of PCAS patients who received $\mathrm{TH}$ at 14 Japanese hospitals. Patients were registered between January 2005 and December 2012. The study was conducted in accordance with the ethical guidelines for epidemiological studies and was approved by the ethics committee of the National Cerebral and Cardiovascular Center. Ethical review boards at all 14 participating centres approved the study protocol.

\section{Patients}

Patients satisfying the following criteria were eligible for registration: (1) all patients who received $\mathrm{TH}$ at any of the 14 participating facilities with successful return of spontaneous circulation (ROSC) after out-of-hospital cardiac arrest; (2) patients aged $\geq 18$ years; (3) stable hemodynamics after ROSC, including stabilisation by drugs or assisted circulation, such as intra-aortic balloon pumping or percutaneous cardiopulmonary support; (4) persistent coma (Japan coma scale score of 200 or 300, or Glasgow coma scale score of 3-5) after ROSC; and (5) presumed cardiac aetiology of cardiac arrest according to the Utstein guidelines [8]. Patients were excluded for the following reasons: pregnancy, aortic dissection, pulmonary embolism, drug addiction, and poor daily activity before the onset of cardiac arrest.

\section{Treatment}

TH was performed with sedation and analgesia according to the hospital's established procedures. In some cases, ice-cold intravenous fluid was administered over 30-60 min to initiate hypothermia. Hypothermia was initiated and/or maintained by (1) surface cooling with a cooling blanket (Blanketrol II; CSZ Medical, Cincinnati, $\mathrm{OH}$, USA) or a cooling device with self-adhesive, hydrogel-coated pads (Arctic Sun; Medivance, Louisville, KY, USA), or by (2) blood cooling with an extracorporeal direct blood cooling device (KTEK-III, Kawasumi, Tokyo, Japan) or an endovascular cooling device (CoolGard 3000; Alsius, Irvine, CA, USA). Mild hypothermia $\left(32-35{ }^{\circ} \mathrm{C}\right.$ ) was maintained for $24-72 \mathrm{~h}$. Rewarming was conducted slowly and gradually over at least 24$72 \mathrm{~h}$. The hypothermia protocol was conducted according to each institution's protocols.

\section{Study outcomes and statistical analysis}

The patients were divided into two groups according to the target temperature as either $<34{ }^{\circ} \mathrm{C}\left(32.0-33.5{ }^{\circ} \mathrm{C}\right.$; low temperature, Group L) or $\geq 34{ }^{\circ} \mathrm{C}\left(34.0-35.0{ }^{\circ} \mathrm{C}\right.$; moderate temperature, Group M).

The patient's neurological outcome was assessed using the Glasgow-Pittsburgh Cerebral Performance Category (CPC), which included the following five categories: CPC 1 (good recovery), CPC 2 (moderate disability), CPC 3 (severe disability), CPC 4 (vegetative state), and CPC 5 (death) [9]. In the present study, a favourable primary outcome was defined as CPC $1-2$ on day 30.

About cooling method, the duration of time at the target temperature is the duration $(\mathrm{h})$ of keeping the target temperature; rewarming time is the duration (days) with which rewarming starts to reach $36.0{ }^{\circ} \mathrm{C}$; Over-cooling is defined as less than $0.5{ }^{\circ} \mathrm{C}$ from target temperature.

The proportion of patients with a favourable neurological outcome was compared between the two groups for all patients and for specified subgroups of patients using univariate and multivariate analyses with propensity score analysis. In the present study, age and resuscitation interval were eligible factors for the subgroup, which were thought of as global factors having influence for the outcome; 60 years old and 30 min were treated as cut-off values from the median of the previous cohort study [10]. The following subgroups of patients were analysed: age $\leq 60$ vs. $>60$ years and resuscitation interval (interval from collapse to ROSC) of $\leq 30 \mathrm{vs}$. $>30 \mathrm{~min}$. 
Univariate analyses were performed using the MannWhitney $U$ test and Fisher's exact test as appropriate. Propensity score analysis was performed by taking into account age, sex (male), bystander cardiopulmonary resuscitation (CPR), cardiogenic cardiac arrest, and resuscitation interval using the inverse probability of the treatment-weighting (IPTW) method. All statistical analyses were considered significant at $P<0.05$.

Statistical analyses were performed using SPSS software version 16.0 (IBM, Armonk, NY, USA) for all analyses, except for propensity score analysis, which was conducted using $\mathrm{R}$ software version 3.1.2 (GNU general public licence).

\section{Results}

The registry included 467 patients, but the target temperature was not stated for ten patients. The characteristics of the analysed patients are summarised in Table 1. Age, target temperature, maintenance of the target temperature, and the rewarming process were significantly different between Groups L and M.

Table 2 compares the proportions of patients with a favourable neurological outcome between Groups L and $\mathrm{M}$ for all patients and in each subgroup. The proportion of patients with a favourable outcome was significantly different between Groups $\mathrm{L}$ and $\mathrm{M}$ in the subgroup of patients with a resuscitation interval of $\leq 30 \mathrm{~min}$ ( $88 \%$ $(n=24)$ vs. $64 \%(n=281)$ odds ratio (OR) 3.867 (1.12613.286), $P=0.024$ ) [OR; odds ratio (95\% confidence interval)].

Table 3 shows the characteristics of patients in Groups $\mathrm{L}$ and $\mathrm{M}$ in the subgroup of patients with a resuscitation interval of $\leq 30 \mathrm{~min}$. The age in the background showed significant difference in this subgroup (50 vs. 62 years old).

Table 4 shows the results of the propensity score analysis comparing the proportion of patients with a favourable neurological outcome between Groups L and $M$. This analyses shows that the proportion of patients with a favourable neurological outcome was significantly different between Groups $\mathrm{L}$ and $\mathrm{M}$ in the subgroup of patients with a resuscitation interval of $\leq 30 \mathrm{~min}$ (OR; 7.438 (1.769-31.272), $P=0.007$ ).

Table 5 compares the proportion of patients with a favourable neurological outcome in the subgroup of patients with a resuscitation interval of $\leq 30 \mathrm{~min}$. As shown in this table, the proportion of patients was significantly different between Groups $\mathrm{L}$ and $\mathrm{M}$ for the overall subgroup and for patients with ventricular fibrillation/ ventricular tachycardia (VF/VT) $(88 \%(n=24)$ vs. $64 \%$

Table 1 Patient characteristics

\begin{tabular}{|c|c|c|c|}
\hline Variable & $\begin{array}{l}\text { Group L } \\
\left(32.0-33.5^{\circ} \mathrm{C}\right) \\
n=42\end{array}$ & $\begin{array}{l}\text { Group M } \\
\left(34.0-35.0^{\circ} \mathrm{C}\right) \\
n=425\end{array}$ & $P$ value \\
\hline Age (years) & $52(44-61)$ & $61(53-69)$ & $<0.001$ \\
\hline Male (\%) & $37(88 \%)$ & $349(82 \%)$ & 0.399 \\
\hline Bystander CPR (\%) & $18(43 \%)$ & $225(53 \%)$ & 0.257 \\
\hline Witness (\%) & 27/34 (79\%) & 298/373 (80 \%) & 1.000 \\
\hline Cardiogenic cardiac arrest (\%) & $41(98 \%)$ & $398(94 \%)$ & 0.497 \\
\hline VFNT (\%) & $35 / 42(83 \%)$ & $286 / 419$ (68 \%) & 0.052 \\
\hline Target temperature $\left({ }^{\circ} \mathrm{C}\right)$ & $33.0(33.0-33.0)$ & $34.0(34.0-34.0)$ & $<0.001$ \\
\hline Over-cooling (\%) & $15 / 42(36 \%)$ & 86/404 (21\%) & 0.051 \\
\hline Interval from collapse to ROSC (min) & $24(15-41)$ & $21(13-34)$ & 0.154 \\
\hline Interval from ROSC to target temperature (min) & $180(105-290)$ & $170(74-335)$ & 0.657 \\
\hline Duration of time at the target temperature $(\mathrm{h})$ & $34(24-50)$ & $25(24-38)$ & 0.004 \\
\hline Rewarming time (days) & $3(2-4)$ & $2(1-3)$ & $<0.001$ \\
\hline \multicolumn{4}{|l|}{ Cooling method } \\
\hline Initial infusion of cold fluid & $27 / 42(64 \%)$ & $229 / 425$ (54 \%) & 0.255 \\
\hline Surface cooling & $27 / 42(64 \%)$ & 197/416 (47 \%) & 0.051 \\
\hline \multicolumn{4}{|l|}{ Device used } \\
\hline Percutaneous cardiopulmonary support & $6 / 30(20 \%)$ & $84 / 325(26 \%)$ & 0.661 \\
\hline CPC 1-2 on day 30 & 27 (64 \%) & $232(55 \%)$ & 0.258 \\
\hline
\end{tabular}

Values are presented as the $n$ (\%) or median (interquartile range)

$C P R$ cardiopulmonary resuscitation, VF ventricular fibrillation, $V T$, ventricular tachycardia, $R O S C$ return of spontaneous circulation, $C P C$ cerebral performance category 
Table 2 Proportions of patients with favourable neurological outcomes according to the target temperature of therapeutic hypothermia

\begin{tabular}{|c|c|c|c|c|c|}
\hline Variable & Target temperature & $n$ & CPC $1-2$ on day 30 & OR $(95 \% \mathrm{Cl})$ & $P$ value \\
\hline \multirow[t]{2}{*}{ All patients } & $<34^{\circ} \mathrm{C}$ & 42 & $27(64 \%)$ & $1.490(0.770-2.881)$ & 0.258 \\
\hline & $\geq 34^{\circ} \mathrm{C}$ & 424 & $232(55 \%)$ & & \\
\hline \multicolumn{6}{|l|}{ Age } \\
\hline \multirow[t]{2}{*}{$\leq 60$ years } & $<34{ }^{\circ} \mathrm{C}$ & 30 & $21(70 \%)$ & $1.167(0.507-2.685)$ & 0.836 \\
\hline & $\geq 34^{\circ} \mathrm{C}$ & 204 & $136(67 \%)$ & & \\
\hline \multirow[t]{2}{*}{$>60$ years } & $<34^{\circ} \mathrm{C}$ & 12 & $6(50 \%)$ & $1.292(0.404-4.131)$ & 0.769 \\
\hline & $\geq 34^{\circ} \mathrm{C}$ & 220 & $96(44 \%)$ & & \\
\hline \multicolumn{6}{|c|}{ Resuscitation interval } \\
\hline \multirow[t]{2}{*}{$\leq 30 \min$} & $<34^{\circ} \mathrm{C}$ & 24 & $21(88 \%)$ & $3.867(1.126-13.286)$ & 0.024 \\
\hline & $\geq 34{ }^{\circ} \mathrm{C}$ & 281 & $181(64 \%)$ & & \\
\hline \multirow[t]{2}{*}{$>30 \mathrm{~min}$} & $<34^{\circ} \mathrm{C}$ & 14 & $3(21 \%)$ & $0.678(0.178-2.586)$ & 0.756 \\
\hline & $\geq 34^{\circ} \mathrm{C}$ & 115 & 33 (29 \%) & & \\
\hline
\end{tabular}

Values are presented as the $n(\%)$

$C P C$ cerebral performance category, $O R$ odds ratio, $\mathrm{Cl}$ confidence interval

$(n=281)$ OR; $3.867(1.126-13.286), P=0.024$ and $95 \%$ $(n=21)$ vs. $71 \%(n=185)$ OR; $8.244(1.079-62.975), P=$ 0.017 , respectively).

Table 6 shows the results of the propensity score analysis to compare the proportion of patients with a favourable neurological outcome between Groups L and $M$ in a subgroup of patients with an interval of $30 \mathrm{~min}$ from collapse to ROSC and VF/VT. The difference in the proportion of patients with a favourable neurological outcome was statistically significant between Groups L and $M$ in this subgroup of patients (OR; 19.43 (2.39158.04), $P=0.006)$.

\section{Discussion}

In the present study, $\mathrm{TH}$ with a lower target temperature of $32.0-33.5{ }^{\circ} \mathrm{C}$ (i.e. Group L) significantly improved the proportion of patients with favourable neurological outcomes in the subgroup of patients with a resuscitation interval of $\leq 30 \mathrm{~min}$, as compared with $\mathrm{TH}$ with a higher target temperature of $34.0-35.0{ }^{\circ} \mathrm{C}$ (i.e. Group $\mathrm{M}$ ) (Table 2). However, it must be acknowledged that with the background characteristics of Groups L and M, the age in the background showed significant difference in this subgroup (50 vs. 62 years old) (Table 3). Therefore, propensity score analysis with the IPTW method was

Table 3 Characteristics of patients with a resuscitation interval of $\leq 30 \mathrm{~min}$

\begin{tabular}{|c|c|c|c|}
\hline \multirow[t]{3}{*}{ Variables } & Group L & Group M & \multirow{3}{*}{$\begin{array}{l}P \\
\text { value }\end{array}$} \\
\hline & $\left(32.0-33.5^{\circ} \mathrm{C}\right)$ & $\left(34.0-35.0^{\circ} \mathrm{C}\right)$ & \\
\hline & $n=24$ & $n=281$ & \\
\hline Age (years) & $50(38-57)$ & $62(53-69)$ & $<0.001$ \\
\hline Male (\%) & $19(79 \%)$ & $228(81 \%)$ & 0.789 \\
\hline Bystander CPR (\%) & $9(38 \%)$ & $146(52 \%)$ & 0.205 \\
\hline Witness (\%) & $17 / 21(81 \%)$ & 205/256 (80 \%) & \\
\hline Cardiogenic cardiac arrest (\%) & $23(96 \%)$ & $263(70 \%)$ & 1.000 \\
\hline VFNT (\%) & $21 / 24(88 \%)$ & 185/277 (67 \%) & \\
\hline Target temperature $\left({ }^{\circ} \mathrm{C}\right)$ & $33.0(32.0-33.0)$ & $34.0(34.0-34.0)$ & $<0.001$ \\
\hline Over-cooling (\%) & $7 / 24(29 \%)$ & $54 / 270(20 \%)$ & \\
\hline Interval from collapse to ROSC (min) & $17(11-23)$ & $15(10-22)$ & 0.469 \\
\hline Interval from ROSC to target temperature (min) & $194(165-324)$ & $180(95-360)$ & 0.460 \\
\hline Duration of time at the target temperature (h) & $34(24-49)$ & $24(24-38)$ & 0.013 \\
\hline Rewarming time (days) & $3(2-4)$ & $2(1-3)$ & $<0.001$ \\
\hline CPC 1-2 on day 30 (\%) & $21(88 \%)$ & $181(64 \%)$ & 0.024 \\
\hline
\end{tabular}

Values are presented as the $n$ (\%) or median (interquartile range)

$C P R$ cardiopulmonary resuscitation, VF ventricular fibrillation, $V T$ ventricular tachycardia, $R O S C$ return of spontaneous circulation, $C P C$ cerebral performance category 
Table 4 Comparison of the proportion of patients with a favourable neurological outcome between the two target temperature groups using propensity score analysis with the inverse probability of the treatment-weighting method

\begin{tabular}{|c|c|c|c|}
\hline Variable & Target temperature & OR $(95 \% \mathrm{Cl})$ & $P$ value \\
\hline All patients & $<34^{\circ} \mathrm{C}$ (vs. $\left.\geq 34^{\circ} \mathrm{C}\right)$ & $1.381(0.596-3.197)$ & 0.452 \\
\hline \multicolumn{4}{|l|}{ Age } \\
\hline$\leq 60$ years & $<34^{\circ} \mathrm{C}$ (vs. $\left.\geq 34^{\circ} \mathrm{C}\right)$ & $0.779(0.297-2.147)$ & 0.657 \\
\hline$>60$ years & $<34^{\circ} \mathrm{C}$ (vs. $\left.\geq 34^{\circ} \mathrm{C}\right)$ & $3.812(0.945-15.367)$ & 0.061 \\
\hline \multicolumn{4}{|c|}{ Resuscitation interval } \\
\hline$\leq 30 \min$ & $<34^{\circ} \mathrm{C}$ (vs. $\left.\geq 34^{\circ} \mathrm{C}\right)$ & 7.438 (1.769-31.272) & 0.007 \\
\hline$>30 \mathrm{~min}$ & $<34{ }^{\circ} \mathrm{C}$ (vs. $\left.\geq 34{ }^{\circ} \mathrm{C}\right)$ & $0.583(0.145-2.344)$ & 0.449 \\
\hline
\end{tabular}

The propensity score analysis incorporated the following variables: age, sex (male), bystander cardiopulmonary resuscitation, cardiogenic cardiac arrest, and resuscitation interval

$O R$ odds ratio, $\mathrm{Cl}$ confidence interval

applied, and confirmed the significant difference in the outcome between Groups L and M in this subgroup of patients (Table 4).

We also found a significant difference in the VF/VT ratio between Group L and M ( $88 \%$ vs. $67 \%$ ) in patients with a resuscitation interval of $\leq 30 \mathrm{~min}$. Therefore, to confirm that the effectiveness of lower target temperature $\mathrm{TH}$ was independent of the VF/VT ratio, we compared the outcomes between VF/VT and non-VF/VT cases, because VF/VT cases tend to have better outcomes than non-VF/VT cases [10]. In VF/VT cases, we found that the lower target temperature $\mathrm{TH}$ conferred better neurological outcomes, but there was no difference between the two groups of patients in non-VF/VT cases (Tables 5 and 6).

The TTM study included two groups of patients with target temperatures of 33 and $36^{\circ} \mathrm{C}$. The characteristics of both groups were similar in terms of their mean age (33 vs. $36{ }^{\circ} \mathrm{C}$; 64 vs. 64 years old, respectively), proportion of males (83 vs. $79 \%$, respectively), proportion of patients receiving bystander CPR (73 vs. $73 \%$, respectively), proportion of patients with VF/VT (73 vs. $73 \%$, respectively), and mean interval from collapse to ROSC ( 25 vs. $25 \mathrm{~min}$, respectively) [3]. Comparing the TTM study and our study, it is apparent that our study population was younger, and bystander CPR was less frequent, but the resuscitation interval was similar.

There were some differences between our study and the TTM study, including shorter time to reach the target temperature (our study vs. TTM study; 180 vs. about $480 \mathrm{~min}$ ), longer time at the target temperature (34 vs. about $20 \mathrm{~h}$ ), and longer rewarming period (3 days vs. 9 h). Several animal studies have demonstrated potential beneficial outcomes of rapid cooling [11-13]; prolonged TH may reduce secondary brain damage [14]; and slow rewarming may attenuate traumatic brain injury [15-18]. Therefore, it seems that these TH methods improved the neurological outcomes of PCAS patients.

Therefore, there might be a possibility that the fundamental cooling method (prolonged $\mathrm{TH}$ and long rewarming period) had an advantage for outcome. Actually, the favourable outcome of the present study (Group L; $64 \%$ and Group M; $55 \%$ ) could be superior to the outcome of TTM study $\left(33{ }^{\circ} \mathrm{C}\right.$ group; $46 \%$ and $36{ }^{\circ} \mathrm{C}$ group; $48 \%$ ). This topic should be discussed in a further study.

Based on the results of animal studies, it is thought that lower target temperature $\mathrm{TH}$ could improve the neurological outcomes compared with higher target

Table 5 Proportions of patients with favourable neurological outcomes according to the target temperature of therapeutic hypothermia in patients with a resuscitation interval of $\leq 30 \mathrm{~min}$

\begin{tabular}{|c|c|c|c|c|c|}
\hline Variable & Target temperature & $n$ & CPC 1-2 on day 30 & OR $(95 \% \mathrm{Cl})$ & $P$ value \\
\hline \multirow[t]{3}{*}{ Resuscitation interval $\leq 30 \mathrm{~min}$} & $<34{ }^{\circ} \mathrm{C}$ & 24 & 21 (88 \%) & $3.867(1.126-13.286)$ & 0.024 \\
\hline & $\geq 34{ }^{\circ} \mathrm{C}$ & 281 & 181 (64\%) & & \\
\hline & $<34{ }^{\circ} \mathrm{C}$ & & 20 (95 \%) & $8.244(1.079-62.975)$ & 0.017 \\
\hline \multirow[t]{2}{*}{ VFNT } & $\geq 34^{\circ} \mathrm{C}$ & 21 & $131(71 \%)$ & & \\
\hline & $<34{ }^{\circ} \mathrm{C}$ & 185 & 1 (33 \%) & $0.479(0.042-5.465)$ & 0.617 \\
\hline \multirow[t]{2}{*}{ Non-VFNT } & $\geq 34{ }^{\circ} \mathrm{C}$ & 3 & $47(51 \%)$ & & \\
\hline & & 92 & & & \\
\hline
\end{tabular}


Table 6 Comparison of the proportion of patients with a favourable neurological outcome between the two target temperature groups using propensity score analysis with the inverse probability of the following treatment-weighting method: patients with a resuscitation interval of $\leq 30 \mathrm{~min}$ and ventricular fibrillation/ventricular tachycardia

\begin{tabular}{llll}
\hline Variable & Target temperature & OR $(95 \% \mathrm{Cl})$ & $P$ value \\
\hline VFNT & $<34^{\circ} \mathrm{C}\left(\right.$ vs. $\left.\geq 34^{\circ} \mathrm{C}\right)$ & $19.43(2.39-158.04)$ & 0.006 \\
\hline
\end{tabular}

The propensity score analysis incorporated the following variables: age, sex (male), bystander CPR, and resuscitation interval

$O R$ odds ratio, $C l$ confidence interval

temperatures [4-6]. However, clinical studies have not yet shown similar effects of lower target temperature $\mathrm{TH}$ in humans. In the present study, we found that patients with a resuscitation interval of $\leq 30 \mathrm{~min}$ had better outcomes following lower target temperature $\mathrm{TH}$, and this shorter resuscitation interval might mimic the design of animal studies. Irrespective of the reason, it seems that lower target temperature $\mathrm{TH}$ might be advantageous in patients with a shorter resuscitation interval.

Extracorporeal cooling is currently applied in the clinical setting, and it is vital that the target temperature is reached within $20 \mathrm{~min}$ after starting cooling [19]. A recent clinical study showed that a combination of $\mathrm{TH}$ with rapid cooling and a cardiopulmonary support device improved the neurological outcomes compared with conventional methods, when performed about $60 \mathrm{~min}$ after VF/VT cardiac arrest. [20] However, it is not clear whether rapid cooling and/or the support device contributed to the improved outcomes. It is possible that quicker cooling could improve the outcome and expand the induction period of $\mathrm{TH}$. In the present study, in which induction cooling was achieved within $180 \mathrm{~min}$, a significant difference between the two target temperatures was only noted for patients with a resuscitation interval of $\leq 30 \mathrm{~min}$.

This study has some limitations to mention. First, we performed subgroup analyses of an observational study. Therefore, the results should be confirmed in future studies. Second, several cooling methods were used, but any advantages of the cooling methods could not be shown in this study. Third, the effects of the cooling protocol were not analysed in sufficient detail because of differences in the maintenance and rewarming processes between the two groups of patients. Fourth, neurological outcome was assessed on day 30, which might not be enough observation periods.

\section{Conclusions}

Although lower target temperature TH for PCAS patients has not been established, including indications, methods, and procedures, the present results might indicate that lower target temperature $\mathrm{TH}$ could be candidate for patients with a resuscitation interval of $\leq 30 \mathrm{~min}$. We conclude that these results warrant further studies examining the effects of lower target temperature $\mathrm{TH}$ in PCAS patients.

\author{
Abbreviations \\ CPC: Glasgow-Pittsburgh cerebral performance category; OR: odds ratio; \\ PCAS: post-cardiac arrest syndrome; PS: propensity score; ROSC: return of \\ spontaneous circulation; TH: therapeutic hypothermia; TTM: target temperature \\ management; VFNT: ventricular fibrillation/ventricular tachycardia.
}

\section{Competing interests}

The authors declare that they have no competing interests.

\section{Authors' contributions}

All authors conceived and designed the study, wrote the study protocol, and contributed to clinical data acquisition. TK performed statistical analyses and wrote the first draft of the manuscript. All authors revised and commented on the manuscript and approved the final version.

\section{Acknowledgements}

We thank all of the members of the J-PULSE-Hypo study group who participated in this multicenter observational study: Department of Cardiovascular Medicine, Division of Cardiovascular Care Unit, National Cerebral and Cardiovascular Center, Suita, Osaka; Department of Cardiology, Cardiopulmonary Resuscitation and Emergency Cardiovascular Care, Surugadai Nihon University Hospital, Tokyo; Emergency and Critical Care Center, Sapporo City University Hospital, Sapporo; Advanced Critical Care and Emergency Center,

Yokohama City University Medical Center, Yokohama; Emergency Medicine, Osaka Mishima Emergency and Critical Care Center, Osaka; Emergency and Critical Care Medicine Center, Osaka City General Hospital, Osaka; Division of Cardiology, Osaka Police Hospital, Osaka; Senri Critical Care Medical Center, Saiseikai Senri Hospital, Osaka; Department of

Cardiology, Sumitomo Hospital, Osaka; Emergency and Critical Care Center, Kagawa University Hospital, Kagawa; Advanced Medical Emergency and Critical Care Center, Yamaguchi University Hospital, Ube; Division of Cardiology, Kokura Memorial Hospital, Kokura; and Department of Epidemiology and Biostatistics, National Center of Neurology and Psychiatry, Tokyo, Japan.

\section{Grant}

A research grant for Cardiovascular Disease (H19-Shinkin-03; The study for the establishment of the prehospital system in acute myocardial infarction and stroke) from the Ministry of Health, Labour, and Welfare, Japan.

\section{Author details}

${ }^{1}$ Emergency and General Medicine, Kumamoto University Hospital, 1-1-1 Honjo, Chuo-ku, Kumamoto 860-8556, Japan. ${ }^{2}$ Advanced Medical Emergency and Critical Care Center, Yamaguchi University Hospital, Ube, Japan. ${ }^{3}$ Senri Critical Care Medical Center, Saiseikai Senri Hospital, Osaka, Japan. ${ }^{4}$ Division of Cardiovascular Care Unit, Department of Cardiovascular Medicine, National Cerebral and Cardiovascular Center, Suita, Japan. ${ }^{5}$ Department of Emergency Medicine, Sapporo Medical University School of Medicine, Sapporo, Japan. ${ }^{6}$ Department of Cardiology, Hiroshima City Hospital, Hiroshima, Japan. ${ }^{7}$ Division of Cardiology, Kokura Memorial Hospital, Kokura, Japan. ${ }^{8}$ Emergency Medicine, Osaka Mishima Emergency and Critical Care Center, Osaka, Japan. ${ }^{9}$ Emergency and Critical Care Medical Center, Osaka City General Hospital, Osaka, Japan. ${ }^{10}$ Cardiovascular Division, Osaka Police Hospital, Osaka, Japan. ${ }^{11}$ Department of Emergency and Critical Care Medicine, Chiba Hokusoh Hospital, Nippon Medical School, Inzai, Japan. ${ }^{12}$ Emergency and Critical Care Center, Kagawa University Hospital, Kagawa, Japan. ${ }^{13}$ Department of Cardiology, Sumitomo Hospital, Osaka, Japan. ${ }^{14}$ Department of Psychopharmacology, National Center of Neurology and Psychiatry, Tokyo, Japan. ${ }^{15}$ Department of Cardiology, Cardiopulmonary Resuscitation and Emergency Cardiovascular Care, Surugadai Nihon University Hospital, Tokyo, Japan. ${ }^{16}$ Shizuoka General Hospital, Shizuoka, Japan.

Received: 13 April 2015 Accepted: 10 June 2015

Published online: 18 June 2015

\section{References}

1. Hypothermia after Cardiac Arrest Study Group. Mild therapeutic hypothermia to improve the neurological outcome after cardiac arrest. N Engl J Med. 2002;346:549-56 
2. Bernard SA, Gray TW, Buist MD, Jones BM, Silvester W, Gutteridge G, et al. Treatment of comatose survivors of out-of-hospital cardiac arrest with induced hypothermia. N Engl J Med. 2002;346:557-63.

3. Nielsen N, Wetterslev J, Cronberg T, Erlinge D, Gasche Y, Hassaqer C, et al. Target temperature management at $33^{\circ} \mathrm{C}$ versus $36{ }^{\circ} \mathrm{C}$ after cardiac arrest. N Engl J Med. 2013;369:2197-206.

4. Li Y, Ristagno G, Guan J, Barbut D, Bisera J, Weil MH, et al. Preserved heart rate variability during therapeutic hypothermia correlated to $96 \mathrm{~h}$ neurological outcomes and survival in a pig model of cardiac arrest. Crit Care Med. 2012;40:580-6.

5. Wang S, Wang S, Li C. Infusion of $4{ }^{\circ} \mathrm{C}$ normal saline can improve the neurological outcome in a porcine model of cardiac arrest. J Trauma Acute Care Surg. 2012;72:1213-9.

6. Ye S, Weng Y, Sun S, Chen W, Wu X, Li Z, et al. Comparison of the durations of mild therapeutic hypothermia on outcome after cardiopulmonary resuscitation in the rat. Circulation. 2012;125:123-9.

7. Yokoyama H, Nagao K, Hase M, Tahara Y, Hazui H, Arimoto H, et al. Impact of therapeutic hypothermia in the treatment of patients with out-of-hospital cardiac arrest from the J-PULSE-Hypo study registry. Circ J. 2011;75:1063-70.

8. Cummins RO, Chamberlain DA, Abramson NS, Allen M, Baskett PJ, Becker L, et al. Recommended guidelines for uniform reporting of data from out-of-hospital cardiac arrest: The Utstein style: a statement for health professionals from a task force of the American Heart Association, the European Resuscitation Council, the Heart and Stroke Foundation of Canada, and the Australian Resuscitation Council. Circulation. 1991;84:960-75.

9. Jennett $\mathrm{B}$, Bond $\mathrm{M}$. Assessment of outcome after severe brain damage. Lancet. 1975;1:480-4

10. Hayakawa K, Tasaki O, Hamasaki T, Sakai T, Shiozaki T, Nakagawa Y, et al. Prognostic indicators and outcome prediction model for patients with return of spontaneous circulation from cardiopulmonary arrest: the Utstein Osaka project. Resuscitation. 2011;82:874-80.

11. Kuboyama K, Safar P, Radovsky A, Tisherman SA, Stezoski SW, Alexander H. Delay in cooling negates the beneficial effect of mild resuscitative cerebral hypothermia after cardiac arrest in dogs: a prospective, randomized study. Crit Care Med. 1993;21:1348-58.

12. Nozari A, Safer P, Stezoski SW, Wu X, Kostelnik S, Radovsky A, et al. Critical time window for intra-arrest cooling with cold saline flush in a dog model of cardiopulmonary resuscitation. Circulation. 2006;113:2690-6.

13. Abella BS, Zhao D, Alvarado J, Hamann K, Vanden Hoek TL, Becker LB. Intra-arrest cooling improves outcomes in a murine cardiac arrest model. Circulation. 2004;109:2786-91.

14. Bisschos L, van der Hoeven JG, Mollnes TE, Hoedemaekers C. Seventy-two hours of mild hypothermia after cardiac arrest is associated with a lowered inflammatory response during rewarming in a prospective observational study. Crit Care. 2014;18:546. http://ccforum.com/content/18/5/546.

15. Suehiro E, Povlishock JT. Exacerbation of traumatically induced axonal injury by rapid posthypothermic rewarming and attenuation of axonal change by cyclosporine A. J Neurosurg. 2001;94:493-8.

16. Maxwell WL, Watson A, Queen R, Conway B, Russell D, Neilson M, et al. Slow, medium, or fast re-warming following post-traumatic hypothermia therapy? An ultrastructual perspective. J Neurotrauma. 2005;22:873-84.

17. Polderman $\mathrm{KH}$, Tjong Tjin Joe R, Peerderman SM, Vandertop WP, Gribes AR Effects of therapeutic hypothermia on intracranial pressure and outcome in patients with severe head injury. Intensive Care Med. 2002;28:1563-73.

18. Polderman $\mathrm{KH}$. Mechanism of action, physiological effects, and complications of hypothermia. Crit Care Med. 2009;37:S186-202.

19. Testori C, Holzer M, Sterz F, Stratil P, Hartner Z, Moscato F, et al. Rapid induction of mild therapeutic hypothermia by extracorporeal veno-venous blood cooling in humans. Resuscitation. 2013;84:1051-5.

20. Sakamoto T, Morimura N, Nagao K, Asai Y, Yokota H, Nara S, et al. Extracorporeal cardiopulmonary resuscitation versus conventional cardiopulmonary resuscitation in adults with out-of-hospital cardiac arrest: a prospective observational study. Resuscitation. 2014;85:762-8. 